\title{
Estabilidad y cambio del voto por la derecha en Chile a la luz de las elecciones presidenciales del 2009*
}

\author{
Miguel Ángel López V.** \\ Andrés Dockendorff V.*** \\ Pedro L. Figueroa R.****
}

\begin{abstract}
Resumen
El análisis de los resultados de las elecciones que significaron el fin de los gobiernos de la Concertación y la llegada de la centroderechista Coalición por el Cambio al poder muestra la ausencia de un realineamiento general del votante chileno. Ello es relevante, no sólo para explicar la victoria de Sebastián Piñera, sino que también para entender la dinámica de estabilidad y cambio electoral en la democracia chilena post-transicional. A partir del análisis de los resultados electorales obtenidos a nivel de coaliciones, el presente artículo demuestra que una de las características más importantes del proceso electoral en la democracia recuperada ha sido la estabilidad del votante chileno, y que si bien las elecciones del 2009-2010 representaron el fin de veinte años de predominio electoral de la coalición de centroizquierda y la alternancia en el poder, desde un punto de vista teórico se trata de una elección "normal" y no de "comicios críticos", siguiendo la nomenclatura de Converse y V.O. Key. Finalmente, se concluye que no se puede hablar de un cisma o cataclismo electoral, ni de reajustes mayores en las relaciones de poder en el sistema político chileno, como lo refleja la composición del Congreso.
\end{abstract}

Palabras clave: Conducta electoral - elecciones - centroderecha - Chile.

\begin{abstract}
The analysis of the election results in Chile which put an end to the Concertation governments and the arrival to power of the center-right Coalition for Change shows the absence of a general realignment of the Chilean voter. This is relevant not only to explain Sebastian Piñera's victory but also to understand the dynamic of electoral stability and change in the post-transitional Chilean democracy. From the analysis of the electoral results obtained at the coalition level, the present article demonstrates that one of the main characteristics of the electoral process in the recovered democracy has been the stability of the Chilean voter; although the 2009-2010 elections represented the end of 20 years of predominance of the center-left coalition and alternance in power, from a theoretical point of view they are "normal" and not "critical elections", following Converse and V.O. Key's nomenclature. Finally, conclusions show that it is not possible to talk of an electoral split or cataclysm, neither of major readjustments of the power relationships in the Chilean political system, as it is reflected by the Congress composition.
\end{abstract}

Keywords: Electoral behavior - elections - centre-right - Chile.

\footnotetext{
* $\quad$ Artículo presentado en el IX Congreso Chileno de Ciencia Política, "La Ciencia Política del Bicentenario: 200 años de política en Latinoamérica", realizado en Santiago entre el 11 y 13 de noviembre del 2010. Algunas partes de este trabajo fueron presentadas en el V Congreso Latinoamericano de Ciencia Política realizado en Buenos Aires entre el 28 y 30 de julio del 2010.

** PhD en Ciencia Política, University of Essex. Correo electrónico: mlopez@uchile.cl

*** Magíster en Ciencia Política, Universidad de Chile. Correo electrónico: adocken@uchile.cl

**** Magíster (c) en Ciencia Política, Universidad de Chile. Correo electrónico: pfiguero@uchile.cl
} 


\section{INTRODUCCIÓN}

El triunfo electoral del actual presidente de Chile, Sebastián Piñera Echeñique, abanderado de la centroderechista Coalición por el Cambio (ex Alianza por Chile) ${ }^{1}$ en las elecciones de 2009-2010², puso fin a dos décadas de gobiernos de la Concertación de Partidos por la Democracia, coalición de centroizquierda que gobernó el país desde el retorno a la democracia en 1990. Este hecho significó el primer triunfo electoral de la derecha durante ese periodo y el primero de un candidato presidencial de ese sector en más de cincuenta años ${ }^{3}$. La votación alcanzada por la derecha en las últimas elecciones transformó los comicios en elecciones genuinamente competitivas, al obtener los candidatos presidenciales de ambas coaliciones una estrecha diferencia en los resultados de la segunda vuelta celebrada en el mes de enero del 2010, apenas un mes antes del terremoto y maremoto que asolaron al país el 27 de febrero de ese año. En esa ocasión, el candidato de la Coalición por el Cambio obtuvo el 51,61\% de la votación, en tanto que el de la Concertación, el ex presidente Eduardo Frei Ruiz-Tagle, el 48,39\%, es decir, una diferencia de 223.000 votos. En el mismo sentido, más significativos aún fueron los resultados de las elecciones de diputados ${ }^{4}$, donde la Concertación apenas superó a la Coalición por un estrecho margen de poco más de 60.000 votos (algo así como menos de dos votos por mesa) al obtener ambas coaliciones el 44,3 y el 43,44\% de la votación respectivamente, lo que en definitiva significó -sistema electoral binominal mediante- que por primera vez desde el retorno a la democracia el conglomerado de centroderecha obtuviera mayoría en la Cámara de Diputados (58 escaños versus 57 de la Concertación), consolidando de paso a uno de sus partidos (la Unión Demócrata Independiente, UDI) como la mayor bancada en la Cámara Baja.

Un hecho no menor registrado en las últimas elecciones resultó ser, nuevamente, el padrón electoral estancado en algo más de ocho millones de inscritos, lo que sin duda constituye un factor de preocupación señalado tanto por estudiosos como actores políticos. En términos prácticos, esta situación implica un envejecimiento del electorado chileno y la autoexclusión de casi cuatro millones de potenciales electores ${ }^{5}$. Ello, preocupante desde el punto de vista de la participación y legitimidad democrática, ofrece al investigador de la conducta electoral la oportunidad de contar con un marco muestral más o menos estable del electorado a partir del cual poder extraer, a falta de estudios de panel en el país, conclusiones

1 Durante la última campaña electoral la coalición de centroderecha cambió su rótulo tradicional de Alianza por Chile al de Coalición por el Cambio. En este trabajo utilizaremos ambos nombres de manera indistinta. En este mismo sentido, cabe mencionar que este conglomerado ha cambiado en varias oportunidades su denominación electoral, siendo éstas Democracia y Progreso (1989), Unión por el Progreso de Chile (1993), Unión por Chile (1997), Alianza por Chile (2001-2008) y Coalición por el Cambio (a partir de 2009).

2 La primera vuelta de la elección presidencial se realizó en diciembre del 2009 y la segunda o balotaje el 17 de enero del 2010.

3 El último presidente de derecha electo en Chile fue Jorge Alessandri Rodríguez, quien gobernó entre 1958 y 1964.

4 Las elecciones del 2009 fueron concurrentes para presidente (primera vuelta), diputados y la mitad de los senadores.

5 En Chile ha existido un sistema de inscripción voluntaria y voto obligatorio, que ha sido modificado durante el año 2011. Hasta ese entonces, el número de no inscritos ha sido estimado en 3.855.000 personas, de las cuales más del 70\% corresponde a jóvenes entre 18 y 19 años de edad. 
e inferencias acerca de su comportamiento. No obstante, la argucia metodológica que -de manera apócrifa- podría posibilitar dicha consecuencia no buscada del estancamiento del padrón no impide reiterar aquí lo preocupante de esta situación, la que se ha transformado en una de las constantes del sistema político chileno.

En este trabajo estudiaremos las elecciones presidenciales del 2009 en su primera y segunda vuelta a la luz de la estabilidad y cambio del votante chileno. Después de más de veinte años de elecciones y con un padrón electoral relativamente fijo, sostenemos que la conducta electoral del votante chileno ha alcanzado gran estabilidad. Considerando la estabilidad sostenida, este trabajo estimará la movilidad del voto entre las elecciones de diputados y presidenciales, así como también la movilidad del voto entre la primera y segunda vuelta de la última elección presidencial, intentando responder las preguntas que interrogan acerca del patrón de votación por la derecha y, en términos más específicos, de dónde provinieron los votos que dieron el triunfo al candidato de la derecha, Sebastián Piñera.

A continuación será presentada la discusión teórica sobre el estudio de la conducta electoral y de las elecciones en tanto factor de estabilidad o cambio de los apoyos electorales a partir de la categorización utilizada en los modelos de las elecciones críticas, de alineación y/o realineamientos del electorado. Luego intentaremos responder la interrogante sobre el origen de los votos de la derecha en la última elección presidencial. Para ello utilizamos el método de inferencia ecológica con la finalidad de estimar los mecanismos de transferencia de votación ocurridos en esa elección. Finalmente, presentamos algunas conclusiones que confirman la estabilidad del voto en Chile.

\section{LA DISCUSIÓN SOBRE EL ESTUDIO DE LA CONDUCTA ELECTORAL Y DE LAS ELECCIONES}

El estudio de la conducta electoral ha sido abordado por tres enfoques principales: el sociológico, el de la identificación partidaria (ambos centrados en las influencias de largo plazo) y el modelo de la elección racional (asociado a los factores de corto plazo). Dentro de los determinantes de largo plazo, muy vinculados a la estabilidad, destacan los sociales y religiosos (Lazarsfeld et al., 1944; Lipset y Rokkan, 1967; Clark y Lipset, 1991; Franklin et al., 1992). Para el modelo sociológico, el sufragio se relaciona con los niveles en los que diferentes grupos (sociales, religiosos, geográficos u otros) votan por determinados partidos y la forma en que los patrones electorales están estrechamente asociados a la posición que esos grupos tienen en la sociedad (Lazarsfeld et al., 1944). Las tendencias de voto según clases sociales constituyen el ejemplo clásico de este enfoque, donde los partidos de derecha representarían los intereses de las clases más acomodadas, los de izquierda los intereses de los trabajadores, y los de centro los de las clases medias.

El modelo de identificación partidaria estudia el voto desde una perspectiva más individual, donde el elector es quien desarrolla una identificación psicológica duradera con un sector o partido (Campbell et al., 1960). Según este modelo, la identificación partidaria 
sería adquirida inicialmente desde la familia, luego sería reforzada por la pertenencia a ciertos grupos y por el voto por un partido determinado a través del tiempo.

Cuando las influencias de largo plazo dejan de ser importantes al momento de explicar la conducta electoral, ya sea por el declive de las divisiones de clases (Clark y Lipset, 1991; Franklin et al., 1992) o por la caída en los niveles de identificación partidista (Dalton et al., 1984; Crewe y Denver, 1985), las variables de corto plazo adquieren mayor relevancia. El modelo de la elección racional explica el voto a partir del supuesto de electores racionales que sufragan por el partido o candidato que mejor representa sus intereses (Enelow y Hinich, 1984). Dentro de los factores de corto plazo que operan en la decisión, la economía ocupa un lugar preponderante. En términos ideales, el Ilamado voto económico es concebido como la expresión en las urnas de las preferencias sobre el correcto manejo de la economía del gobierno de turno. En concreto, los votantes evalúan al gobierno incumbente sobre la base de los resultados de las políticas económicas: inflación, desempleo, crecimiento, valor de la moneda, entre otros (Dorussen y Palmer, 2002). Además del voto económico, las características personales del candidato (King, 2002) han adquirido gran preponderancia como factor explicativo de la conducta electoral, cuestión estrechamente asociada al decaimiento de los niveles de identificación partidaria ${ }^{6}$.

Respecto al estudio de las elecciones propiamente tales, la pregunta general que se hacen los politólogos es acerca de su significado en tanto factor de estabilidad o cambio de los apoyos electorales. Sobre este tema la literatura especializada nos presenta diversos modelos de análisis y paradigmas, como el de las elecciones críticas y de alineación y/o realineamientos del electorado. Así como la teoría de los clivajes electorales (Lipset y Rokkan, 1967) ha sido por muchos años el principal marco teórico en el estudio de la estabilidad de los votantes en Europa, la teoría de las elecciones críticas (Key, 1955) ha sido una de las teorías más utilizadas para explicar la estabilidad y el cambio electoral en Estados Unidos. Para Key, una elección crítica consiste en un tipo de elección de amplia participación e intensidad que produce grandes reajustes en las relaciones de poder, dando origen a coaliciones o grupos electorales estables (Key, 1955: 4). Si bien en Chile durante el último tiempo sólo el plebiscito de 1988 cumpliría estas condiciones, los sucesivos aportes realizados a la teoría harían plausible su aplicación en el caso chileno.

En la búsqueda de una clasificación de las elecciones presidenciales en Estados Unidos, Campbell et al. (1960) retomaron los estudios de Key (1955) y agregaron a su análisis los conceptos de elecciones de "mantención", "desviación" y "realineamiento". Considerando la estabilidad electoral del votante norteamericano en base a la identificación partidaria, los autores introdujeron la clasificación para explicar el mantenimiento y el cambio electoral en el tiempo. Bajo este enfoque, las elecciones de mantención son aquellas que perpetúan el statu quo de apoyos electorales a los partidos de una elección a otra, mientras que en las

6 La crisis de los partidos se ha instalado con fuerza en el debate académico de las últimas décadas. Este diagnóstico crítico se sostiene en la constatación de la desintegración de las organizaciones partidistas, la pérdida de influencia de los partidos en los gobiernos, la erosión de la militancia y el debilitamiento de la identificación partidaria entre los ciudadanos (Fiorina, 2002; Clarke y Stewart, 1998). 
elecciones de "desviación" las lealtades electorales no se ven seriamente alteradas, pero las actitudes de voto llevan a la derrota del partido mayoritario. Después de que desaparecen los actores o eventos que desviaron esas fuerzas de lo normal/esperado, el balance político regresa al nivel que más cercanamente representa las divisiones subyacentes de la identificación partidaria. "Una elección de desviación es entonces un revés temporal que ocurre en un periodo cuando uno u otro partido mantiene una clara ventaja en las preferencias de largo plazo del electorado" (Campbell et al., 1960: 275). En tanto, las elecciones de realineamiento corresponden a la clasificación de elecciones críticas de Key (1955), las que según los autores citados son bastante poco frecuentes y están asociadas a periodos de gran crisis. Pomper (1967) agrega una cuarta clasificación, la de elección de "conversión", que es aquella donde, a pesar de que la mayor parte de la base de apoyo del partido mayoritario está en proceso de cambio, éste sigue ganando las elecciones.

Evans y Norris (1999) retoman esta teoría y la aplican a las elecciones británicas clasificándolas en tres tipos: de mantención, de des-alineamiento y de re-alineamiento. Las segundas a su vez se subdividen en elecciones de "desviación" (idénticas a la categoría establecida por Campbell et al., 1960) y de "des-alineamiento secular" (muy similares a las elecciones de conversión de Pomper, 1967). Las elecciones de re-alineamiento se dividen en "seculares" (el paulatino fortalecimiento de la votación hacia un partido determinado) y las ya mencionadas elecciones críticas.

De todas las clasificaciones mencionadas, la de elección crítica ha sido la más difundida y utilizada en la literatura. Desde un punto de vista histórico tienen amplias repercusiones, ya que permiten ordenar y periodizar elecciones (Weatherford, 2002). En ciencia política, la teoría de las elecciones críticas introdujo una visión de tipo más bottom-up en el estudio de la conducta electoral. En las teorías de realineamiento y en la elección crítica son los votantes, y no la élite o los partidos, el componente dinámico del cambio electoral (Nardulli, 1995).

Siendo en términos generales las continuidades menos atractivas que el cambio, las elecciones de mantención y de desviación han sido menos estudiadas y teóricamente han sido conceptualizadas como de "voto normal" (Converse, 1966). El voto normal se refiere a una representación de la estabilidad en el voto partidario y en el apoyo electoral de un partido de una elección a la otra. Para Converse (1969), un país alcanza estabilidad en su apoyo electoral a los partidos e identificación partidaria después de dos o tres generaciones. La estabilidad en el apoyo partidario resulta ser de especial importancia en América Latina debido a que la lealtad de los votantes hacia un partido determinado constituye un requisito imprescindible para la institucionalización de los sistemas de partidos (Mainwaring y Scully, 1995).

\section{EL VOTO POR LA DERECHA EN CHILE}

A partir del análisis de los datos de opinión pública y los resultados electorales de los comicios de 1999, algunos análisis comenzaron a hablar de una "derechización" del electorado, dado un cambio en la conducta electoral de las mujeres, la clase media y los nuevos inscritos (Lehmann y Hinzpeter, 2001). ¿Se soporta esta tesis en la evidencia 
proporcionada por las elecciones posteriores? ¿Constituye la elección de 2009-2010 la consolidación de esa derechización del electorado chileno iniciada a fines de los noventa?

Dentro de un marco general de estabilidad electoral, la derecha ha dado cuenta, en las elecciones legislativas, de un notable crecimiento de uno de sus partidos, fruto de una estrategia electoral de largo plazo que comenzó a rendir frutos en 1999, en la disputada elección Lagos-Lavín, y consolidada en 2001, cuando la UDI se convierte en el partido más votado del país, desplazando al Partido Demócrata Cristiano (PDC). ¿Qué explica este último fenómeno?

En términos generales, la estabilidad es un rasgo dominante en el escenario electoral chileno, lo que, en perspectiva comparada, se traduce en indicadores de volatilidad electoral extremadamente moderados, en especial para América Latina. En veinte años no han surgido nuevos partidos relevantes, y los partidos existentes en 1989 siguen siendo competitivos dos décadas después, lo que vale en especial para la derecha, que ha mostrado un menor faccionalismo que otros partidos, como el PDC y el Partido Socialista (PS), en los últimos años. Esta dinámica de estabilidad en el apoyo electoral de más de una década ha estado fuertemente influenciada por el legado del plebiscito de 1988 por el que Pinochet fue obligado a convocar a elecciones libres el año siguiente. Este plebiscito tuvo las características de una elección fundacional ${ }^{7}$ (Drake y Jaksic, 1995), ya que configuró las dos más importantes coaliciones de partidos de la actualidad y produjo un alineamiento electoral que se mantiene hasta nuestros días ${ }^{8}$. Dado que la Concertación se mantuvo en el poder desde el regreso a la democracia por dos décadas, y no se ha producido gran volatilidad entre los electores, las primeras elecciones chilenas post 1988 constituyen elecciones de mantención (Evans y Norris, 1999), reforzadas además por un sistema electoral que incentiva la competencia intracoaliciones ${ }^{9}$. Sin embargo, el alza en la votación por la Alianza, sobre todo por la UDI en las elecciones de diputados del 2001, podría ser considerada como una elección de desviación (Evans y Norris, 1999). Ello ya que se produjo un revés temporal en el patrón "normal" de votos sobre todo influenciados por la personalidad del líder de la Alianza de entonces, Joaquín Lavín. Luego, en las elecciones presidenciales de 2009-2010, se produce una reorganización del escenario electoral chileno con la llegada al poder de la coalición de centroderecha luego de veinte años de predominio de la Concertación.

Esta notable estabilidad electoral, no alterada mayormente a nivel parlamentario por los resultados del 2009, y la continuidad de las etiquetas partidarias coexiste con niveles

7 A pesar de que O'Donnell y Schmitter (1986: 62) consideran elección fundacional a la primera elección competitiva y multipartidaria que sigue a un periodo autoritario, el plebiscito de 1988 tuvo muchas de las características de una elección fundacional, sobre todo su capacidad para congelar el sistema de partidos y la relativa estabilidad que produce en las preferencias del electorado.

8 Regresiones simples, usando los resultados del plebiscito de 1988 como variable independiente, muestran que ésta es una excelente predictora de los resultados en elecciones posteriores, especialmente en el caso de las elecciones de diputados.

9 El sistema electoral chileno, elaborado por el autoritarismo, elige dos escaños por circunscripción, pero para obtener ambos escaños el partido más votado debe doblar en votos a su más cercano contendor. Esto facilita la competencia en coaliciones y la concentración de los votos. 
decrecientes de identificación partidaria, baja confianza en los partidos, petrificación del padrón electoral y un desencanto general con la política. El viejo electorado sigue siendo estable al votar por partidos y coaliciones, a pesar de que paradojalmente no se reconoce identificado o cercano con esos partidos en las encuestas (López, 2008). Esta alta institucionalización partidista y estabilidad electoral no se explicaría en función de la estrecha relación entre partidos y sociedad civil, sino más bien debido a los resultados artificiales producidos por la institucionalidad electoral (Luna, 2008).

Hasta aquí se ha explorado descriptivamente la evolución electoral de los partidos de la derecha en la democracia recuperada. Pero ¿qué factores explican el voto por la derecha chilena en el post-autoritarismo? En términos generales, existen dos grandes corrientes de opinión en la literatura sobre la evolución de las preferencias electorales de los chilenos. Por un lado están quienes defienden la idea de continuidad (Scully y Valenzuela, 1993; Siavelis, 2000; Valenzuela, 1995). En otra vereda están quienes postulan que la experiencia autoritaria y el apoyo o rechazo al régimen militar habría generado un nuevo eje de división política y electoral (Torcal y Mainwaring, 2003; Tironi y Agüero, 1999; Tironi et al., 2001; Agüero et al., 1998).

La coalición de centroderecha habría obtenido mejores votaciones en los sectores alto y bajo (de acuerdo a los indicadores de desarrollo humano -IDH- disponibles por comuna), mientras que la Concertación tendría mejores resultados en unidades comunales de IDH medio (Altman, 2004). Por otra parte, el análisis de la conducta electoral empleando las categorías ocupacionales muestra que los "trabajadores" han votado de forma consistente por la Concertación. No obstante, en las elecciones presidenciales 1999-2000 los "trabajadores no calificados" votaron con mayor fuerza por el candidato de la centroderecha (Joaquín Lavín) que los "trabajadores calificados" (López, 2004b).

El Gráfico 1 muestra la evolución de la conducta electoral de los chilenos cruzada por las características socioeconómicas de los electores en las elecciones a la Cámara Baja entre 1997 y 2005, a partir de la información proporcionada por encuestas de opinión.

El gráfico muestra la erosión de los factores socioeconómicos como explicativos de la conducta electoral en Chile. Como muestran los datos de opinión pública, el voto de los sectores más acomodados por los partidos de derecha disminuye de más de un 50\% en 2001 a poco más de un cuarenta en la última elección de diputados. Los patrones para los sectores medio y bajo son más inestables, y si bien el apoyo de los sectores medio y bajo a la Concertación supera al apoyo de los mismos grupos a la Alianza, da la impresión de que las elecciones del 2001 son las menos "clasistas" de todas, ya que los niveles de respaldo de ambos grupos sociales a ambas coaliciones son más cercanos.

\section{El voto por la derecha en las elecciones de diputados de 2009 y la estabilidad del voto en Chile}

En las elecciones generales de diciembre del 2010, junto con las elecciones presidenciales se renovó por completo la Cámara de Diputados (120 escaños) y la mitad de la Cámara 


\section{GRÁFICO 1}

El voto según características socioeconómicas en elecciones de diputados (1997-2005)

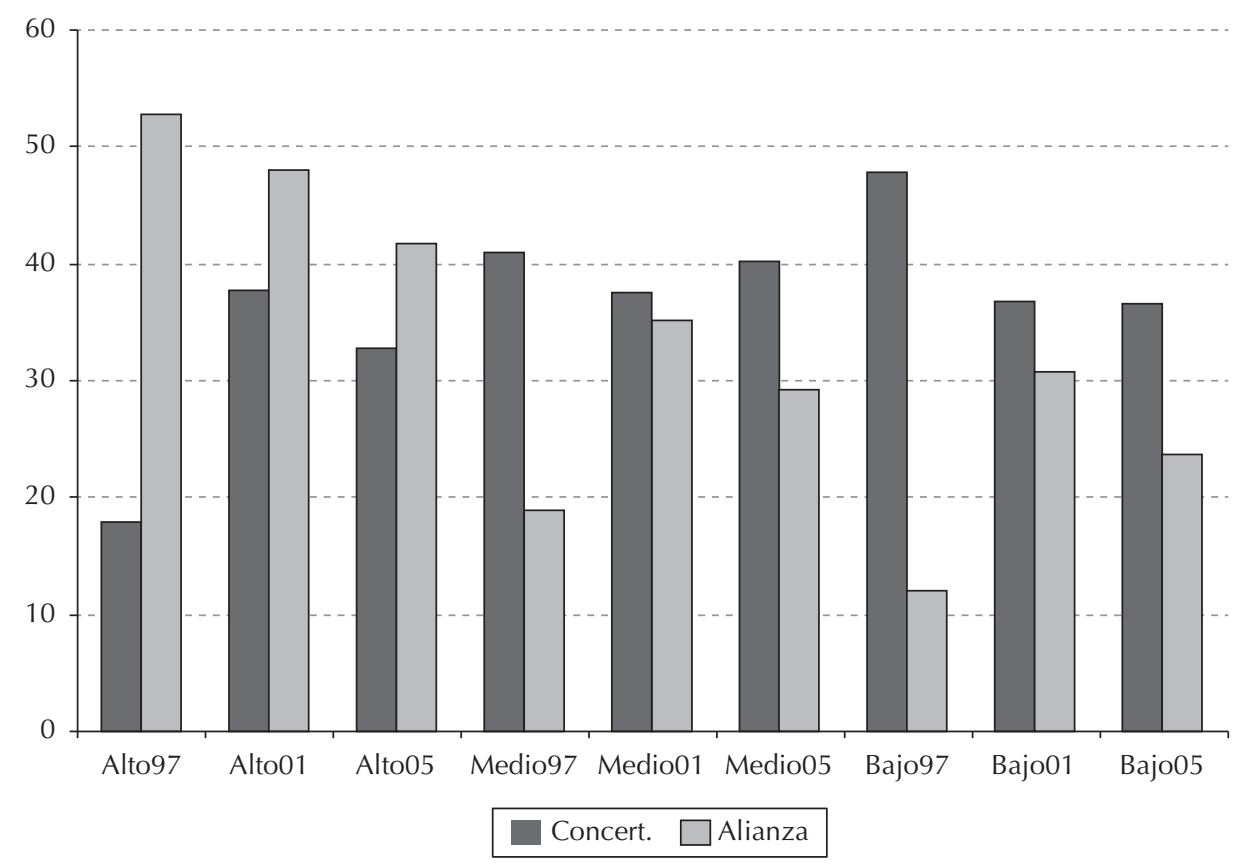

Fuente: Elaborado a partir de los datos disponibles en www.cepchile.cl

Alta. Como se mencionó anteriormente, la Concertación se impuso con un mínimo margen de 44,36\% de los votos sobre el 43,44\% obtenido por la Coalición por el Cambio. Sin embargo, dadas las características del sistema electoral binominal chileno, la coalición de centroderecha eligió 58 diputados y la Concertación 57. Entre los elementos más destacados de esta elección está el retorno al Congreso, por primera vez desde 1973, de tres diputados del Partido Comunista (PC), quienes formaban parte de la lista de la Concertación. Sin duda, el gran ganador de las elecciones de la Cámara Baja fue la UDI, partido de derecha que negoció cupos en las elecciones de la Cámara Baja con su socio electoral, Renovación Nacional (RN), para apoyar a Sebastián Piñera en las elecciones presidenciales. Dados los efectos del sistema electoral binominal, con sólo un 23,04\% de los votos la UDI obtuvo 37 diputados, casi un tercio de la Cámara.

Una de las mejores formas de estudiar la estabilidad del votante chileno durante los últimos veinte años de democracia es a través del análisis de los resultados de las elecciones de diputados. La Tabla 1 muestra los resultados obtenidos por las coaliciones y los partidos en las seis elecciones de diputados realizadas durante el periodo. En ella se observa que, en promedio, la Concertación ha obtenido un 50,2\% de las preferencias mientras que la Coalición por el Cambio (ex Alianza) sólo un 38,9\%, no obstante haber obtenido mayor presencia en la Cámara debido a los efectos del sistema binominal. 
TABLA 1

Resultado elecciones Cámara de Diputados (1989-2009)

\begin{tabular}{|l|c|c|c|c|c|c|}
\hline & 1989 & 1993 & 1997 & 2001 & 2005 & 2009 \\
\hline ALIANZA (1) & 34,25 & 33,48 & 35,89 & 44,32 & 38,72 & 43,43 \\
\hline Renovación Nacional (RN) & 18,28 & 16,31 & 16,77 & 13,76 & 14,12 & 17,82 \\
\hline Unión Demócrata Independiente (UDI) & 9,82 & 12,11 & 14,45 & 25,19 & 22,36 & 23,04 \\
\hline Otros & 0,06 & 0,25 & & 0,06 & & 0,27 \\
\hline Independientes & 6,09 & 4,81 & 4,67 & 5,31 & 2,24 & 2,30 \\
\hline CONCERTACIÓN & 50,50 & 55,40 & 50,52 & 47,88 & 51,76 & 42,32 \\
\hline Partido Demócrata Cristiano (PDC) & 25,99 & 27,12 & 22,98 & 18,92 & 20,76 & 14,24 \\
\hline Partido Por la Democracia (PPD) & 11,45 & 11,84 & 12,55 & 12,72 & 15,42 & 12,65 \\
\hline Partido Socialista (PS) & & 11,93 & 11,05 & 10 & 10,05 & 9,90 \\
\hline Partido Radical Socialdemócrata (PRSD) & 3,94 & 3,77 & 3,13 & 4,05 & 3,54 & 3,78 \\
\hline Independientes & 9,12 & 0,74 & 0,81 & 2,19 & 1,99 & 1,75 \\
\hline JUNTOS PODEMOS & 5,37 & 6,00 & 9,79 & 6,35 & 7,40 & 3,46 \\
\hline Partido Humanista (PH) & 0,99 & 1,01 & 2,91 & 1,13 & 1,56 & 1,44 \\
\hline Partido Comunista (PC) & $4,38 *$ & 4,99 & 6,88 & 5,22 & 5,14 & $2,02 * *$ \\
\hline Independientes & 6,46 & 3,32 & 1,88 & 1,51 & 2,11 & $10,76 * * *$ \\
\hline Otros partidos & & & & & 0,7 & \\
\hline
\end{tabular}

Fuente: www.elecciones.gov.cl

(1) En la última elección la Alianza se inscribió bajo el rótulo de Coalición por el Cambio.

* En 1989 los humanistas integraron la lista de la Concertación y los comunistas la lista PAIS.

** En 2009 el PC integró la lista de la Concertación, por lo tanto su 2,02\% se suma al voto de la lista A en los últimos comicios parlamentarios.

*** El Partido Regionalista de los Independientes (PRI) se presentó junto al Movimiento Amplio Social (MAS), obteniendo un $4,56 \%$ y un 5,40\%, respectivamente, lo que explica que la categoría "Otros Partidos" en 2009 alcance el porcentaje que se indica. 
Durante el periodo analizado, una de las constantes observadas es que los partidos de la Concertación siempre han obtenido más votos que los de su principal contrincante, siendo la última elección y la del 2001 las más reñidas y competitivas. Las variaciones más significativas se observan a nivel de partidos, donde se pueden apreciar periodos de auge y decadencia de algunos conglomerados y la persistente estabilidad de otros. En la Coalición de centroderecha, RN y la UDI presentan una media similar de 16,8 y $17,8 \%$ respectivamente. Sin embargo, a pesar de un leve ascenso en la última elección, el partido del presidente, $\mathrm{RN}$, ha perdido votación en el tiempo, mientras que la UDI se ha fortalecido. Entre los partidos que forman la Concertación, el PDC mantiene en promedio el mayor promedio de votos, con el 21,7\%, sin embargo su votación ha experimentado considerables erosiones Ilegando a un $14,24 \%$ en la última elección, a pesar de que el candidato presidencial de la Concertación (Eduardo Frei Ruiz-Tagle) pertenecía a ese partido. En tanto, la votación por el PPD y por el PS se ha mantenido estable, variando alrededor del 12,8 y 8,8\%. La formación más pequeña de la Concertación, el PRSD, también mantiene estable su apoyo electoral, aunque en torno a un exiguo y testimonial 3,7\%.

Esta dinámica de estabilidad en el apoyo electoral de más de una década fue fuertemente influenciada por el plebiscito de 1988, que tuvo las características de una elección fundacional (Drake y Jaksic, 1995), ya que configuró las dos más importantes coaliciones de partidos que persisten en la actualidad y originó un alineamiento electoral que se mantiene vigente. Esta estabilidad a nivel de coaliciones y partidos no implica necesariamente una estabilidad a nivel de votantes, los que pueden cambiar sus intenciones de voto más allá de lo que las medidas de nivel macro, como el índice de volatilidad electoral (Pedersen, 1979), podrían reflejar. Estudios a nivel de votante usando inferencia ecológica (López, 2004a) o encuestas con preguntas retrospectivas (Huneeus, 2002) muestran que sobre el $60 \%$ de los electores tiende a votar por el mismo partido de una elección a otra. A pesar de que la volatilidad es mayor que a nivel agregado, los resultados confirman la persistencia en la actitud del votante chileno.

La Tabla 2 muestra los resultados de las elecciones presidenciales chilenas a partir del retorno a la democracia, en cuyos inicios los partidos de derecha eran más frecuentemente asociados con el régimen militar y los excesos de la dictadura, en especial la UDI que, como vimos anteriormente, en la primera elección del periodo apenas alcanzó un 9\%. En tal contexto, la Concertación se impuso ampliamente y en primera vuelta en las elecciones presidenciales de 1989 y 1993, luego de las cuales se comenzaron a estrechar las diferencias, forzando, a partir de la elección de 1999, la segunda vuelta electoral hasta el triunfo del 2010. En los comicios de 2005, si bien la oposición superó a la Concertación al sumar los votos de sus candidatos (Joaquín Lavín y Sebastián Piñera) en primera vuelta, éstos no fueron traspasados totalmente en el balotaje. Como dijimos anteriormente, la estabilidad electoral del voto presidencial se rompió el 2009, al obtener el candidato de la Concertación, Eduardo Frei, sólo el 29,6\% en la primera vuelta y Piñera la mayoría relativa, con un 44,05\%.

Se observa un aumento en el apoyo hacia el sector conforme se aleja el periodo dictatorial. Incluso, el relativo éxito electoral de Lavín en 1999 estuvo asociado a una estrategia de "despinochetización" de su campaña en medio de la detención del ex dictador 
TABLA 2

Resultado elecciones presidenciales (1989-2009)

\begin{tabular}{|l|c|c|c|c|c|c|c|c|}
\hline $\begin{array}{c}\text { COALICIÓN DE } \\
\text { LOS CANDIDATOS }\end{array}$ & 1989 & 1993 & $19991^{\mathrm{a}}$ & $19992^{\mathrm{a}}$ & $20051^{\mathrm{a}}$ & $20052^{\mathrm{a}}$ & $20091^{\mathrm{a}}$ & $20092^{\mathrm{a}}$ \\
\hline Alianza & 29,4 & 24,41 & 47,51 & 48.69 & $48,64^{*}$ & 46,5 & 44,05 & 51,6 \\
\hline Indep. de derecha & 15,43 & 6,18 & - & - & - & - & - & - \\
\hline Total derecha & 44,83 & 30,59 & 47,51 & 48.69 & 48,64 & 46,5 & 44,05 & 51,6 \\
\hline Concertación & 55,17 & 57,98 & 47,96 & 51.31 & 45,96 & 53,5 & 29,6 & 48,39 \\
\hline PC & - & 4,7 & 3,19 & - & - & - & 6,21 & - \\
\hline PH-Verde & - & 1,17 & 0,51 & - & 5,4 & - & - & - \\
\hline Indep. de izquierda & - & 5,55 & 0,44 & - & - & - & - & - \\
\hline Independientes & - & - & 0,38 & - & - & - & 20,13 & - \\
\hline
\end{tabular}

Fuente: www.elecciones.gov.cl

*El 2005 la Alianza fue representada por Joaquín Lavín (23,23\%) y Sebastián Piñera (25,41\%).

en Londres. Por su parte, Piñera logró obtener la victoria en un contexto donde los temas de la dictadura no constituían issues efectivos de campaña y varios años después de la muerte de Pinochet.

\section{IV. ¿DE DÓNDE OBTUVO LOS VOTOS LA CENTRODERECHA? (O ¿A DÓNDE SE FUERON LOS VOTOS DE LA CONCERTACIÓN?)}

Si el candidato presidencial de la Concertación (E. Frei Ruiz Tagle) hubiese obtenido una votación similar a la de los diputados de la Concertación, o si hubiese logrado recuperar al menos dos tercios de los votantes del candidato Enríquez-Ominami ${ }^{10}$, es probable que la Concertación haya retenido el gobierno por un quinto periodo. El objetivo principal de los estudios de desviación o mantenimiento electoral es analizar el sentido del traspaso de votos y los niveles de fidelidad electoral de una elección a otra. En segundo lugar, si efectivamente se produce un realineamiento, indagar sobre las bases sociales o partidarias del fenómeno. Para responder estas interrogantes, el instrumento metodológico más adecuado son las encuestas pre o post-electorales de panel, las que desafortunadamente no existen

10 Los candidatos presidenciales que participaron en la primera vuelta electoral fueron Eduardo Frei (Concertación), Sebastián Piñera (Coalición por el Cambio), Marco Enríquez-Ominami (candidato independiente proveniente de la Concertación) y Jorge Arrate (Juntos Podemos). 
en Chile. Ante esta situación, una alternativa de análisis válida metodológicamente es la inferencia ecológica, que permite realizar análisis a escala de datos agregados a través de su transformación en datos individuales evitando incurrir en la llamada "falacia ecológica". Al realizar este procedimiento, los datos adquieren las características de una encuesta de panel.

Usando el método de inferencia ecológica de Thomsen ${ }^{11}$ (1987), estimamos cómo los votantes en la elección de diputados del 2009 votaron en las elecciones presidenciales del mismo año, cuántos de ellos se mantuvieron leales a los candidatos de la misma coalición y cuántos votaron cruzado. Además, estimamos la movilidad electoral entre las dos vueltas presidenciales para conjeturar hacia dónde se fueron los votos de los candidatos Jorge Arrate y Marco Enríquez-Ominami.

En la Tabla 3 se estima la distribución porcentual de los votos presidenciales (filas) a partir de la votación obtenida por las coaliciones en las elecciones de diputados (columnas) realizadas de manera concurrente. Allí se observa que del total de votantes que apoyaron a candidatos de la Concertación en las elecciones de diputados, el 53\% de ellos apoyaron también a Frei en las presidenciales. En cambio, sólo un 15\% de ellos apoyó a Piñera en primera vuelta; 17,8\% a Enríquez-Ominami (MEO); 4,8\% a Arrate y un 8,5\% no emitió un voto válido o se abstuvo.

Los resultados observados muestran una gran fidelidad de voto de los sufragantes por la Alianza/Coalición por el Cambio, que alcanza casi al $80 \%(79,7)$ y un menor apoyo al candidato presidencial Eduardo Frei entre quienes votaron por la Concertación (sólo un 53,8\%). Como señalan Campbell et al. (1960), considerando los resultados generales de las elecciones presidenciales (Tabla 2) y la incapacidad de trasferir el apoyo parlamentario al candidato presidencial (Tabla 3), esta se trataría de una elección presidencial de "desviación" más que un cambio crítico en los patrones electorales. Los autores mencionan que se deben a hechos o personas específicas las que producen este swift de votantes, en este caso la débil candidatura de Frei y la irrupción del candidato descolgado de la Concertación, Marcos Enríquez-Ominami.

En la Tabla 4 presentamos la estimación de las fidelidades de los votantes de la Cámara Baja por partidos, para proyectar el apoyo que los votantes de cada conglomerado le dieron al candidato de su coalición. Dado que no todos los partidos presentaron candidatos en todos los distritos, los resultados se estiman por grupos de partidos para así mantener la homogeneidad de los datos, un requisito fundamental en este tipo de análisis de inferencia ecológica. Las características de desviación de la primera vuelta presidencial se acentúan al estimar los apoyos partidarios al mostrar los estragos que provocó la candidatura de

11 El método logit de inferencia ecológica de Thomsen (1987) es una alternativa a las tablas de Goodman (1953, 1959) para estimar datos individuales a partir de datos agregados evitando la falacia ecológica. Conocido también como método de estructuras latentes (Achen y Shively, 1995), se usan las tablas de Goodman para estimar la movilidad de votos o la relación entre votos y datos socioeconómicos usando resultados electorales y datos censales a nivel agregado. A partir de los marginales de la tabla, Thomsen usa correlaciones gamma bajo supuestos de homogeneidad de la data como proxy de correlaciones ecológicas logísticas. 


\section{TABLA 3}

Movilidad y estabilidad electoral en elección presidencial y de diputados 2009 (coaliciones)

\begin{tabular}{|lr|r|r|r|r|c|}
\cline { 2 - 6 } \multicolumn{1}{c|}{} & \multicolumn{4}{c|}{ PRESIDENCIALES 2009 (PRIMERA VUELTA) } & \multirow{2}{*}{ TOTAL } \\
\hline DIPUTADOS 2009 POR COALICIÓN & FREI & PIÑERA & MEO & ARRATE & NUL./BLA./ABS. & \\
\hline Concertación & 53,8 & 15,0 & 17,8 & 4,8 & 8,5 & 100,0 \\
\hline Alianza & 6,5 & 79,7 & 6,4 & 2,5 & 4,9 & 100,0 \\
\hline PH y otros & 5,5 & 15,2 & 48,9 & 16,6 & 13,7 & 100,0 \\
\hline PRI y otros & 24,0 & 9,4 & 38,2 & 7,1 & 21,3 & 100,0 \\
\hline Indep. 2009 & 12,2 & 46,1 & 16,0 & 8,5 & 17,3 & 100,0 \\
\hline Nulos/Blancos 1989 & 14,5 & 6,3 & 45,5 & 12,2 & 21,3 & 100,0 \\
\hline Abstención & 7,5 & 13,3 & 9,1 & 5,2 & 64,9 & 100,0 \\
\hline & 24,8 & 36,9 & 16,9 & 5,2 & 16,1 & 100,0 \\
\hline
\end{tabular}

Fuente: Elaboración propia.

TABLA 4

Movilidad y estabilidad electoral en elección presidencial y de diputados 2009 (partidos)

\begin{tabular}{|l|r|r|r|r|c|c|}
\cline { 2 - 7 } \multicolumn{1}{c|}{} & \multicolumn{4}{c|}{ PRESIDENCIALES 2009 (PRIMERA VUELTA) } & \multirow{2}{*}{ TOTAL } \\
\hline DIPUTADOS 2009 POR PARTIDOS & FREI & PIÑERA & MEO & ARRATE & NUL./BLA./ABS. & \\
\hline DC & 49,9 & 33,3 & 5,6 & 2,3 & 9 & 100,0 \\
\hline PS/PPD/PR & 38,3 & 30,9 & 17,2 & 6,3 & 7,3 & 100,0 \\
\hline Indep. Concert. & 21,2 & 42,1 & 13,4 & 2,1 & 21,2 & 100,0 \\
\hline RN/Chile 1 & 11,7 & 68,3 & 6,3 & 4,4 & 9,3 & 100,0 \\
\hline UDI/Independientes & 20,3 & 53,1 & 15,1 & 3,1 & 8,2 & 100,0 \\
\hline PH & 7,8 & 17,4 & 49,1 & 14,7 & 11,1 & 100,0 \\
\hline PRI & 31,6 & 10,1 & 35,9 & 5,9 & 16,5 & 100,0 \\
\hline Independientes & 16,0 & 49,2 & 14,9 & 7,0 & 12,9 & 100,0 \\
\hline Nulos/Blancos 1989 & 19,9 & 7,1 & 44,6 & 10,5 & 17,9 & 100,0 \\
\hline Abstención & 11,6 & 16,8 & 10,0 & 5,1 & 56,6 & 100,0 \\
\hline & 24,8 & 36,9 & 16,9 & 5,2 & 16,1 & 100,0 \\
\hline
\end{tabular}

Fuente: Elaboración propia. 
Piñera entre los votantes del PDC. Proveniente de una familia tradicional de ese partido, la apelación electoral de Piñera afectó fuertemente a los votantes del centro político chileno, consiguiendo un 33,3\% del voto por la DC. Solamente uno de cada dos votantes $(49,9 \%)$ de la DC en las elecciones de la Cámara Baja apoyó al candidato de la colectividad en la primera vuelta de las elecciones presidenciales. Aparentemente, Frei tampoco agradó mucho a los votantes de los partidos más a la izquierda dentro de la Concertación, ya que los votantes socialistas (PS), los partidarios del PPD y del PC, registraron un bajo apoyo $(38,3 \%)$ al ex presidente. Llama la atención el relativamente alto apoyo que consiguió Piñera en este sector de la Concertación (39,9\%) al igual que el logrado por candidaturas independientes dentro de la Concertación (42,1\%). Si bien en este último caso se trataba de candidatos que no tenían apoyo explícito partidario y no consiguieron grandes porcentajes de votos, en el primero la situación fue diferente. Una hipótesis a considerar emana de la fuerte personalización de las campañas, tanto al Congreso como a la Presidencia, lo que erosiona los apoyos electorales partidarios.

El partido del presidente (RN) cedió algunos cupos a un grupo de ex concertacionistas desencantados que formaron el partido Chile Primero. Los votantes de estos partidos (RN/ Chile $1^{\circ}$ ) mantuvieron su apoyo al candidato RN en las elecciones presidenciales $(68,3 \%)$, mientras que solamente un 11,7\% emigró a Frei.

Una de las mayores incertidumbres durante el periodo de campaña era acerca de la capacidad de Piñera para atraer a los votantes de la UDI, partido con el que el actual presidente había tenido una larga historia de desencuentros y críticas antes de su nominación como candidato de la Coalición por el Cambio. Si bien la mayoría $(53,1 \%)$ de los electores de la UDI votaron por el candidato de derecha, sólo un 20,3\% lo hizo por Frei y un 15,1\% se sintió atraído por la candidatura anti establishment de Enríquez-Ominami.

En la segunda vuelta, y sin las candidaturas alternativas de Enríquez-Ominami y Arrate, Piñera y Frei se enfrentaron en una competencia por los votos y por conseguir los apoyos necesarios para superar el 50\% de los votos válidos. Para Piñera se trataba de conseguir un porcentaje muy pequeño (casi seis puntos porcentuales) pero para Frei el camino era más complejo, ya que requería sumar más de veinte puntos. Dado lo decisivo del acto electoral, los votantes de las elecciones parlamentarias del mes anterior fueron bastante más estables en el apoyo a los candidatos presidenciales. Los votantes por candidatos de la Concertación en las elecciones de diputados se cuadraron fuertemente con Frei, alcanzando un 75,1\% de los votos. Se trató de un avance considerable debido a que un mes antes sólo un 53,8\% lo había apoyado. Sin embargo, desafortunadamente para el candidato de la Concertación, la fidelidad de los votantes de la Coalición por el Cambio fue superior $(85,9 \%)$, lo que sin duda contribuyó al triunfo de Piñera (ver Tabla 5).

Dado que en Chile los niveles de abstención y de votación nula o en blanco son bastante estables, la capacidad de ambos candidatos para atraer votos de los eliminados en la primera vuelta se transformó en uno de los factores clave para obtener el triunfo. Si bien la cantidad de votos que necesitaba Piñera era mucho menor que la de Frei, lo cierto era que se trataba de dos candidatos que en algún momento habían estado en las filas de la 


\section{TABLA 5}

Movilidad y estabilidad electoral en elección presidencial y de diputados 2009 (segunda vuelta/partidos)

\begin{tabular}{|c|c|c|c|c|c|}
\hline \multirow{3}{*}{$\begin{array}{l}\text { DIPUTADOS } 2009 \\
\text { POR COALICIÓN }\end{array}$} & \multirow{2}{*}{\multicolumn{4}{|c|}{ PRESIDENCIALES 2009 (SEGUNDA VUELTA) }} & \\
\hline & & & & & \multirow[b]{2}{*}{ TOTAL } \\
\hline & FREI10 & PIÑERA10 & NUL./BLA.89 & ABSTENCIÓN & \\
\hline Concertación & 75,1 & 18,8 & 1,5 & 4,6 & 100,0 \\
\hline Alianza & 9,8 & 85,9 & 0,8 & 3,4 & 100,0 \\
\hline $\mathrm{PH}$ otros & 35,3 & 35,7 & 10,9 & 18,1 & 100,0 \\
\hline PRI otros & 61,4 & 17,0 & 6,7 & 15,0 & 100,0 \\
\hline Independientes 2009 & 20,5 & 59,1 & 3,5 & 16,9 & 100,0 \\
\hline Nulos/Blancos 1989 & 60,8 & 17,5 & 13,8 & 7,9 & 100,0 \\
\hline \multirow[t]{2}{*}{ Abstención } & 12,4 & 18,8 & 2,4 & 66,4 & 100,0 \\
\hline & 40,6 & 43,2 & 3,0 & 13,3 & 100,0 \\
\hline
\end{tabular}

Fuente: Elaboración propia.

Concertación. La Tabla 6 muestra la gran estabilidad de los votantes que ya habían elegido a Frei $(92,6 \%)$ y Piñera $(96,6 \%)$ en la primera vuelta el mes anterior. En el caso de Frei esto era de esperar dado el bajo porcentaje de votos válidos obtenidos en la primera vuelta, que representaba probablemente al núcleo más duro de su apoyo como candidato presidencial. Más problemático resultaba para Piñera mantener la adhesión de un grupo de más de tres millones de electores. No obstante, la votación que Frei necesitaba para vencer a Piñera se convirtió en un umbral demasiado alto de sobrepasar. A pesar del apoyo personal y no formal que Enríquez-Ominami entregó a Frei, el considerable 68\% de su votación que se traspasó a Frei (alrededor de 900 mil votos) no fue suficiente para detener el avance de Piñera. De manera contraria, y a pesar del apoyo explícito y formal entregado por el candidato de la izquierda Jorge Arrate a Frei, no debe extrañar el bajo nivel de traspaso de votos producido $(42,5 \%)$, toda vez que anteriores estudios de inferencia ecológica muestran que los votantes comunistas generalmente prefieren abstenerse o votar nulo o blanco ante la eventualidad de hacerlo por un candidato de la Concertación ${ }^{12}$.

12 Esto habría ocurrido en las segundas vueltas de las elecciones presidenciales de 1999 y 2005, realizadas en los meses de enero de los años 2000 y 2006. 
132 MIGUEL ÁNGEL LÓPEZ V., ANDRÉS DOCKENDORFF V., PEDRO L. FIGUEROA R.

TABLA 6

Movilidad y estabilidad electoral en elección presidencial 2009

(primera y segunda vuelta)

\begin{tabular}{|l|c|c|c|c|c|}
\cline { 2 - 5 } \multicolumn{1}{c|}{} & \multicolumn{4}{c|}{ PRESIDENCIALES 2009 (SEGUNDA VUELTA) } & \multirow{2}{*}{ TOTAL } \\
\hline PRIMERA VUELTA 2009 & FREI10 & PIÑERA10 & NUL./BLA.89 & ABSTENCIÓN & \\
\hline Frei 2009 & 92,6 & 5,5 & 0,3 & 1,7 & 100,0 \\
\hline Piñera 2009 & 1,6 & 96,6 & 0,1 & 1,7 & 100,0 \\
\hline MEO & 68,0 & 15,3 & 6,6 & 10,1 & 100,0 \\
\hline Arrate & 42,5 & 17,4 & 23,3 & 16,8 & 100,0 \\
\hline Nulos/Blancos 1989 & 60,3 & 16,0 & 9,4 & 14,4 & 100,0 \\
\hline Abstención & 9,4 & 17,4 & 1,4 & 71,8 & 100,0 \\
\hline & 40,6 & 43,2 & 3,0 & 13,3 & 100,0 \\
\hline
\end{tabular}

Fuente: Elaboración propia.

\section{CONCLUSIONES}

Al examinar críticamente los resultados y estimar la movilidad del voto podemos ver que no existe algún tipo de realineamiento general del votante. Este tema es importante no sólo para explicar los resultados de la elección presidencial de 2009 y las razones del triunfo de Piñera, sino también para entender las dinámicas de estabilidad y cambio electoral en una democracia como la chilena. Por otra parte, al analizar los resultados obtenidos a nivel de coaliciones políticas se puede observar que la característica más sobresaliente de las elecciones durante el periodo post-autoritario ha sido la estabilidad de las preferencias de los votantes, lo que sin duda estaría asociado al estancamiento del padrón electoral.

Si bien las elecciones generales del 2009 implicaron el fin del periodo de gobierno de veinte años de la Concertación, el primer triunfo electoral de la derecha desde 1958, y la alternancia en el poder dentro de una democracia en forma, desde un punto de vista estrictamente electoral se trata de una elección "normal" (Converse, 1969) y no de una "crítica" (Key), en tanto, como ha quedado demostrado, no se han producido grandes reajustes en las relaciones de poder al interior del sistema político chileno (salvo el hecho no menor de la alternancia) ni mucho menos se han producido nuevas coaliciones o grupos electorales. La última elección corresponde a una de "desviación", debido a que las lealtades electorales no se vieron seriamente alteradas, aun cuando significaron la derrota del candidato de la Concertación.

En cuanto a los patrones de comportamiento electoral observados y la estabilidad del votante chileno, el revés electoral de la Concertación bien podría ser solamente temporal, 
aun cuando resulta difícil pronosticar un regreso al orden anterior, sobre todo porque no se puede asegurar la presencia de un candidato con más arrastre que el anterior en la Concertación, ni mucho menos aún descartar a priori la emergencia de descolgados en las próximas elecciones. Por su parte, dependerá del desempeño en el gobierno de la derecha la posibilidad de transformar esta elección de desviación en una de realineamiento que inaugure un periodo extenso de permanencia de la derecha en La Moneda.

Un fenómeno mucho más interesante es el que se produce a nivel de los partidos, algunos de los cuales han visto erosionado parte de su apoyo (como la DC) mientras que otros lo han aumentado (como la UDI). En el caso particular de la derecha, se han producido una serie de "desviaciones", unas de tipo intracoalicional que han significado el fortalecimiento de la UDI a expensas de RN y otras de tipo extracoalicional, que han favorecido nuevamente a la UDI, partido que se ha visto beneficiado por una serie de "realineamientos seculares", que han significado el paulatino fortalecimiento de la votación hacia ese partido a través de sucesivas elecciones.

\section{BIBLIOGRAFÍA}

Achen, Cristopher H. y W. Phillips Shively (1995): Cross-Level Inference, University of Chicago Press, Chicago.

Agüero, Felipe, Eugenio Tironi, Eduardo Valenzuela y Guillermo Sunkel (1998): “Votantes, partidos e información política: la frágil intermediación política en el Chile post-autoritario", Revista de Ciencia Política, 19 (2), pp. 159-193.

Altman, David (2004): "Redibujando el mapa electoral chileno: incidencia de factores socioeconómicos y género en las urnas", Revista de Ciencia Política, 14 (2), pp. 49-66.

Campbell, Angus, Philip E. Converse, Warren E. Miller y Donald E. Stokes (1960): The American Voter, Wiley, New York.

Clark, Terry Nichols y Seymour Martin Lipset (1991): "Are Social Classes Dying", International Sociology, 6 (4), pp. 397- 410.

Converse, Philip E. (1966): "The Concept of the Normal Vote", en Angus Campbell, Philip E. Converse, Warren Miller y Donald Stokes (eds.): Elections and the Political Order, Wiley, New York, pp. 9-39.

(1969): “Of Time and Partisan Stability", Comparative Political Studies, 2, pp. 139-171.

Crewe, Ivor y David Denver (eds.) (1985): Electoral Change in Western Democracies: Patterns and Sources of Electoral Volatility, Croom Helm, London.

Dalton, Russell, Scott Flanagan y Paul Beck (eds.) (1984): Electoral Change in Advanced Industrial Democracies, Princeton University Press, Princeton.

Dorussen, Han y Harvey Palmer (2002): "The Context of Economic Voting: An Introduction", en Han Dorussen y Michaell Taylor (eds.): Economic Voting, Routledge, London, pp. 1-14.

Drake, Paul W. e Iván Jaksic (1995): "Introduction: Transformation and Transition in Chile, 1982-1990", en Paul W. Drake e Iván Jaksic: The Struggle for Democracy in Chile. Revised Edition, University of Nebraska Press, Lincoln, pp. 1-17.

Enelow, James y Melvin Hinich (1984): The Spatial Theory of Voting: An Introduction, Cambridge University Press, Cambridge. 


\section{MIGUEL ÁNGEL LÓPEZ V., ANDRÉS DOCKENDORFF V., PEDRO L. FIGUEROA R.}

Evans, Geoffrey y Pippa Norris (eds.) (1999): Critical Elections: British Parties and Voters in Long-Term Perspective, Sage, London.

Franklin, Mark N., Thomas T. Mackie y Henry Valen (eds.) (1992): Electoral Change: Responses to Evolving Social and Attitudinal Structures in Western Countries, Cambridge University Press, Cambridge.

Goodman, Leo A. (1953): "Ecological Regression and the Behavior of Individuals", American Sociological Review, 18, pp. 663-64.

(1959): "Some Alternatives to Ecological Correlation", American Journal of Sociology, 64, pp. 610-625.

Huneeus, Carlos (2002): “¿Dónde se fueron los votantes del PDC?”, Informe 175, disponible en: www. ced.cl/ap/wp-content/uploads/2002/01/175.pdf

Key Jr., Valdimer O. (1955): "A Theory of Critical Elections", Journal of Politics, 17 (1), pp. 3-18.

King, Anthony (ed.) (2002): Leaders' Personalities and the Outcomes of Democratic Elections, Oxford University Press, Oxford.

Lazarsfeld, Paul, Bernard Berelson y Hazel Gaudet (1944): The People's Choice, Columbia University Press, New York.

Lehmann, Carla y Ximena Hinzpeter (2001): “¿Nos estamos derechizando? Análisis sobre la base de resultados electorales y encuestas CEP", Puntos de Referencia, 240, Centro de Estudios Públicos.

Lipset, Seymour Martin y Stein Rokkan (1967): Party Systems and Voter Alignments, Free Press, New York.

López, Miguel A. (2004a): "Chilean Voting Patterns", Tesis doctoral, University of Essex, Colchester, UK.

(2004b): "Conducta electoral y estratos económicos: el voto de los sectores populares en Chile", Política, 43, pp. 285- 298.

(2008): "Conducta electoral en Chile: la paradoja de la estabilidad", trabajo preparado para el IV Congreso Latinoamericano de Ciencia Política-ALACIP, San José de Costa Rica, 5-7 de agosto.

Luna, Juan Pablo (2008): "Partidos políticos y sociedad en Chile. Trayectoria histórica y mutaciones recientes", en Arturo Fontaine, Cristián Larroulet, Jorge Navarrete e Ignacio Walker (eds.): Reforma de los Partidos Políticos en Chile, PNUD, Santiago, pp. 75-124.

Mainwaring, Scott y Timothy Scully (eds.) (1995): Building Democratic Institutions. Party Systems in Latin America, Stanford University Press, Palo Alto.

Nardulli, Peter F. (1995): "The concept of a critical realignment, electoral behavior, and political change", American Political Science Review, 89 (1), pp. 10-22.

Norris, Pippa (1999): Critical citizens. Global support for democratic governance, Oxford University Press, Oxford.

Pedersen, Morgan (1979): "The dynamics of European party systems: changing patterns of electoral volatility", European Journal of Political Research, 7 (1), pp. 1-26.

Pomper, Gerald (1967): "Classification of Presidential Elections", Journal of Politics, 29 (4), pp. 535-566.

Scully, Timothy y Samuel Valenzuela (1993): "De la democracia a la democracia. Continuidad y variaciones en las preferencias del electorado y en el sistema de partidos en Chile", Estudios Públicos, 51, pp. 195-228.

Siavelis, Peter (2000): "Continuidad y cambio en el sistema partidista chileno: Sobre los efectos de transformación de una reforma electoral", Revista de Ciencia Política, 20 (2), pp. 82-102. 
Thomsen, Søren R. (1987): Danish Elections 1920-7. A Logit Approach to Ecological Analysis and Inference, Politica, Arthus.

Tironi, Eugenio y Felipe Agüero (1999): “¿Sobrevivirá el nuevo paisaje político chileno?", Estudios Públicos, 74, pp. 151-168.

Tironi, Eugenio, Felipe Agüero y Eduardo Valenzuela (2001): “Clivajes políticos en Chile: perfil sociológico de los electores de Lagos y Lavín", Perspectivas, 5 (1), pp. 73- 87.

Torcal, Mariano y Scott Mainwaring (2003): "The Political Recrafting of Social Bases of Party Competition: Chile, 1973-95", British Journal of Political Science, 33 (1), pp. 55-84.

Valenzuela, Samuel (1995): "Orígenes y transformaciones del sistema de partidos en Chile", Estudios Públicos, 58, pp. 5-80.

Weatherford, M. Stephen (2002): "After the critical election: presidential leadership, competition and the consolidation of the new deal realignment", British Journal of Political Science, 32 (2), pp. 221-257.

Recibido: 20-01-2011

Aceptado: 04-04-2011 